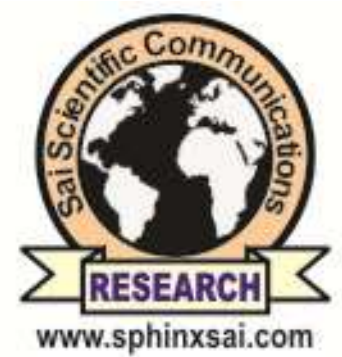

\title{
Simple Thin-Layer Chromatographic and UV-Spectrophotometric Analysis of Promethazine and its N-Demethylation Metabolites from Biological Fluids
}

\author{
Bunu J. Samuel ${ }^{\star}$, Ere Diepreye, and Wilson O. Diana \\ Department of Pharmaceutical and Medicinal Chemistry, Faculty of Pharmacy, Niger \\ Delta University, Wilberforce Island, Bayelsa State, Nigeria. \\ *Correspondence Author: Bunu J. Samuel; \\ Pharmsamuelbunu@gmail.com,+2348069703966
}

\begin{abstract}
Several Ultra-Violet spectrophotometric analytical method has been made available in the analysis of phenothiazine group of drugs but Thin-Layer Chromatographic techniques are not fully utilized. Hence, we were able to develop a succinct, simple and cost-effective TLC and UV-spectrophotometric quantification method for the analysis promethazine and metabolites from biological fluids, validating previous studies. The proposed method was found to be precise, accurate and phenotypic determination and categorization were successfully estimated among the test samples. From the chromatogram intensities, 33.3\% of the study population were classified as poor metabolizers, $40 \%$ were intermediate metabolizers, and $26.67 \%$ extensive metabolizers. Little or no elimination of $\mathrm{N}$-desmethylpromethazine was observed for subjects with poor metabolism in correlation with the severity. Therefore, the phenotypic knowledge will help in the clinical choices as an individual from the same family may likely metabolize a giving drug in the same manner due to their genetic similarities.

Keywords : Promethazine, Metabolites, Thin-Layer Chromatography, cytochrome P450.
\end{abstract}

\section{Introduction}

Promethazine hydrochloride, [N, N-dimethyl-1-(10H-Phenothiazine-10-yl)-propan-2-amine $]$ is a prototype antihistamine among the phenothiazines, that rapidly crosses the blood-brain barrier and its sedative effects of are due to the blockade of histamine $\left(\mathrm{H}_{1}\right)$ receptors in the central nervous system. ${ }^{1}$ It has also displayed strong anticholinergic properties by blocking acetylcholine responses mediated by muscarinic receptors. ${ }^{2}$ The elimination half-life of promethazine in adults ranges from 9 - 16 hours after IV or IM administration and 16-19 hours following oral or rectal administration. The wide range of half-lives reflects the differences in metabolic rate among CYP2D6 genotypes, ranging from ultrarapid to poor metabolizers. ${ }^{3}$ The genetic basis for variation in drug response involves the concept of

Bunu Samuel Jacob et al /International Journal of PharmTech Research, 2020,13(4): 316-324.

DOI: http://dx.doi.org/10.20902/IJPTR.2019.130402 
<smiles>CC(CN1c2ccccc2Sc2ccc(Cl)cc21)N(C)C</smiles>

Promethazine

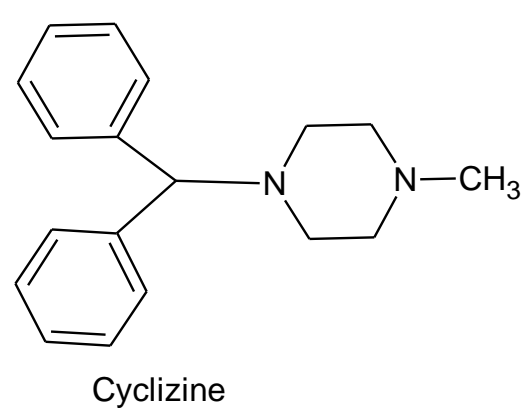

Cyclizine<smiles>CN(C)CCOC(c1ccccc1)c1ccccc1</smiles>

Diphenhydramine

Figure 1: Structures of Promethazine and other antihistamines

pharmacogenetic, which has advanced over the years using genotype to phenotype approach in clinical practice. An individual's response to drugs depends on the complex interaction between environmental, genetic and pharmacokinetic factors. The pharmacokinetics of promethazine involves cytochrome P450 enzymes and genetic variations caused by mutations in the alleles of these enzymes. Thus, suggesting differences in phenotypic polymorphism when administered. ${ }^{4}$ Antihistamines are classified into first and second generations. First-generation antihistamines (promethazine, cyclizine, diphenhydramine, etc.), are non-selective but greater tendencies to cause sedation, while secondgeneration antihistamines (loratadine, terfenadine, fexofenadine, astemizole etc.) are non-sedating and possess less side effects. ${ }^{5}$

Earlier studies have described methods for the extraction of phenothiazines from body tissues based on liquid-liquid extraction (LLE) or solid-phase extraction (SPE), to provide sufficient clean extracts so they can be analyzed by GC/MS or HPLC with optical detection or to reduce matrix effects in methods based on LC/MS/MS. However, analyzing the metabolites of these drugs following oral administration have been limited through methods that involve arduous sample preparation and long chromatographic run times for biological samples. ${ }^{6}$ Several UV spectrophotometric analytical method has been made available in the literature for analyzing some phenothiazine group of drugs but TLC techniques are not fully utilized. ${ }^{7}$ Promethazine sulphoxides have been detected in human plasma following oral administration. ${ }^{8,9}$ These studies suggest that the S-oxidation of promethazine is limited to the gut wall and only occurs during the absorption process. ${ }^{10}$ Hence, we were able to develop a succinct, simple and cost-effective TLC and UV quantification of promethazine and its metabolites from biological fluids following oral administration; a method reported by Ebeshi et al. ${ }^{11}$ and determine the implications on reported adverse drug reactions to provide simple phenotype-based drug therapy.

\section{Materials and Methods}

\section{Reagents}

Two brands of promethazine; Avonum 25mg (Pharmaceutical laboratories, Pakistan), and Sunymine 25mg (Sunymet pharmacy Ltd, Nigeria). Methanol, HCL (JHD, China), 30\% Ammonium hydroxide solution (Loba Chemie, India), n-butanol (Sigma Aldrich, India). All reagents were of analytical grade and used without further purification. 


\section{Study population and Sample Collection}

A survey questionnaire was developed with different sections to distinctively assess the compliance of the subjects to the survey and the possible criteria to which they could be participants of the study with the promise of sole confidentiality. The following categories were included in each section: Section A: Demographics. Section B: Medication and Medical history. Section C: The post medication surveillance, possible adverse effects observed during and after drug administration. Healthy adult individuals from the Niger Delta Area were randomly selected for the study after giving their consent. Thirty subjects including 17 females and 13 males within 18 to 27 years of age were interviewed orally and given questionnaires. They were asked to refrain from alcohol intake, grapefruit and any medication for two weeks before the analysis and during the study. The study was conducted using a parallel single-dose design. Urine samples were collected from the subjects before the administration of Promethazine (25mg). To the first 15 subjects, brand A (Avonum) was administered and post-drug urine samples were collected after 4-24hours. To the other 15 subjects, brand B (Sunymine) was administered and urine was collected at the same time interval. The urine samples were refrigerated at $-20^{\circ} \mathrm{C}$ to avert microbial spoilage and biochemical interactions before analysis.

\section{Standard solution and Preparation of Test Sample}

A stock solution of $5 \mathrm{mg} / \mathrm{ml}$ was prepared in n-butanol from the two standard brands of promethazine. The clear supernatant (organic layer) was carefully separated into a cleaned $10 \mathrm{ml}$ conical flask. One $\mathrm{ml}$ was obtained from each sample and introduced into clean, test tubes, labeled as A \& B (before and after drug administration). To each test tube, $0.3 \mathrm{ml}$ of dil. $\mathrm{HCl}$ was added and vortex for 20 seconds, heated in at $100{ }^{\circ} \mathrm{C}$ for 1.30 minutes and allowed to cool. $4 \mathrm{ml}$ of $\mathrm{n}$-butanol was further added into the solutions and centrifuged for 30minutes. The clear supernatant layer transferred into plain EDTA tubes.

\section{Thin-Layer Chromatographic Analysis}

The TLC analysis was carried out using Merck KGaA, 64271 Darmstadt, Germany while the spectrophotometric measurement was done using Shimadzu UV-1700 UV-VIS lamp. The mobile phase developed using n-butanol and ammonium hydroxide in 10:0.5 ratio. TLC plates were cut into sizes to be used for the analysis, labeled, and heated in the oven for 15 minutes. The test solution of the samples was spotted on the origin TLC plates marked at $1 \mathrm{~cm}$ away from the starting point of the plate using a heparinized capillary tube, allowed to dry and the plates placed the solvent chamber until the solvent gets to the front. The plates were removed from and allowed to evaporate. The dried plates were placed in a UV-Visible spectrophotometric lamb at $254 \mathrm{~nm}$ wavelength and observed for visible spots. The $\mathrm{R}_{\mathrm{f}}$ value for the stock solution and the test solutions were calculated and recorded accordingly respectively. Thereafter, the plates were also placed in an iodine tank to visualize more spots that were not revealed in the UV lamp. The study was approved by the research and ethics committee of the Faculty of Pharmacy, Niger Delta University, Wilberforce Island, Nigeria.

\section{Results}

Results obtained from the analysis are presented in tables and charts. 
Table 1. Gives a summary of the Retardation factor of chromatographic spots on TLC as exhibited by the 30 subjects following oral administration of Avernum (D1-D15) and Sunymine (W16-W30) respectively, demographics and relationship between intensity of spots, phenotype, and ADRs severity of the observed adverse reactions in the study population.

\begin{tabular}{|c|c|c|c|c|c|c|c|c|c|}
\hline \multirow{2}{*}{$\begin{array}{l}\text { Study } \\
\text { Code }\end{array}$} & \multirow[t]{2}{*}{ Gender } & \multirow{2}{*}{$\begin{array}{l}\text { Time } \\
\text { (hrs) }\end{array}$} & \multicolumn{3}{|c|}{$\mathbf{R}_{\mathrm{f}}$ Values } & \multirow[t]{2}{*}{ Ethnicity } & \multirow[t]{2}{*}{ Intensity } & \multirow[t]{2}{*}{ Phenotype } & \multirow[t]{2}{*}{ ADRs } \\
\hline & & & $\mathbf{A}$ & $\mathbf{B}$ & $\mathbf{C}$ & & & & \\
\hline D1 & $\mathrm{F}$ & 12 & 0.81 & - & 0.70 & Ogbia & $\mathrm{L}$ & IM & Mild \\
\hline D2 & $\mathrm{F}$ & 14 & 0.85 & - & 0.74 & Omoku & $\mathrm{L}$ & IM & Moderate \\
\hline D3 & $\mathrm{F}$ & 16 & 0.80 & - & 0.68 & Benin & I & PM & Mild \\
\hline D4 & $\mathrm{F}$ & 12 & 0.89 & - & 0.75 & Ishan & $\mathrm{L}$ & IM & Mild \\
\hline D5 & $\mathrm{F}$ & 15 & 0.85 & - & 0.72 & Etche & $\mathrm{L}$ & IM & No reaction \\
\hline D6 & $\mathrm{F}$ & 8 & 0.86 & - & 0.76 & Calabar & VL & EM & No reaction \\
\hline D7 & $\mathrm{F}$ & 12 & 0.85 & - & 0.75 & Isoko & VL & EM & Mild \\
\hline D8 & $\mathrm{F}$ & 13 & 0.88 & 0.23 & 0.70 & Ijaw & $\mathrm{L}$ & $\mathrm{IM}$ & Mild \\
\hline D9 & M & 8 & 0.85 & - & 0.74 & Kalabari & VL & EM & Mild \\
\hline D10 & $\mathrm{F}$ & 12 & 0.86 & - & 0.69 & Epie & I & PM & Mild \\
\hline D11 & M & 22 & 0.84 & - & 0.66 & Urhobo & I & PM & Severe \\
\hline D12 & M & 10 & 0.83 & - & 0.68 & Calabar & I & PM & Severe \\
\hline D13 & M & 16 & 0.83 & - & 0.71 & Ogbia & I & PM & Moderate \\
\hline D14 & M & 4 & 0.89 & - & - & Efik & I & PM & Mild \\
\hline D15 & M & 9 & 0.88 & - & 0.74 & Ijaw & $\mathrm{L}$ & IM & Moderate \\
\hline W16 & M & 14 & 0.84 & 0.29 & 0.74 & Esan & $\mathrm{L}$ & $\mathrm{IM}$ & Mild \\
\hline W17 & M & 8 & 0.86 & - & 0.75 & Omoku & VL & EM & No reaction \\
\hline W18 & M & 12 & 0.85 & - & 0.69 & Urhobo & I & PM & Severe \\
\hline W19 & $\mathrm{F}$ & 12 & 0.86 & - & 0.71 & Ikwere & $\mathrm{L}$ & $\mathrm{IM}$ & Moderate \\
\hline W20 & $\mathrm{F}$ & 12 & 0.85 & 0.17 & 0.75 & Ijaw & VL & EM & Mild \\
\hline W21 & F & 18 & 0.84 & - & 0.68 & Oron & I & PM & Severe \\
\hline W22 & M & 12 & 0.86 & - & 0.70 & Nembe & $\mathrm{L}$ & IM & No reaction \\
\hline W23 & F & 6 & 0.87 & - & 0.77 & Ijaw & VL & EM & Mild \\
\hline W24 & $\mathrm{F}$ & 12 & 0.87 & - & - & Isoko & VL & EM & Mild \\
\hline W25 & M & 12 & 0.86 & - & 0.74 & Ogoni & $\mathrm{L}$ & IM & Mild \\
\hline W26 & M & 14 & 0.86 & - & 0.69 & Esan & I & PM & Mild \\
\hline W27 & M & 10 & 0.85 & - & 0.68 & Urhobo & I & PM & Severe \\
\hline W28 & $\mathrm{F}$ & 16 & 0.85 & 0.23 & 0.74 & Nembe & $\mathrm{L}$ & IM & Mild \\
\hline W29 & $\mathrm{F}$ & 10 & 0.83 & - & 0.72 & Epie & $\mathrm{L}$ & IM & Moderate \\
\hline W30 & $\mathrm{F}$ & 12 & 0.84 & - & 0.75 & Etan & VL & EM & Mild \\
\hline
\end{tabular}



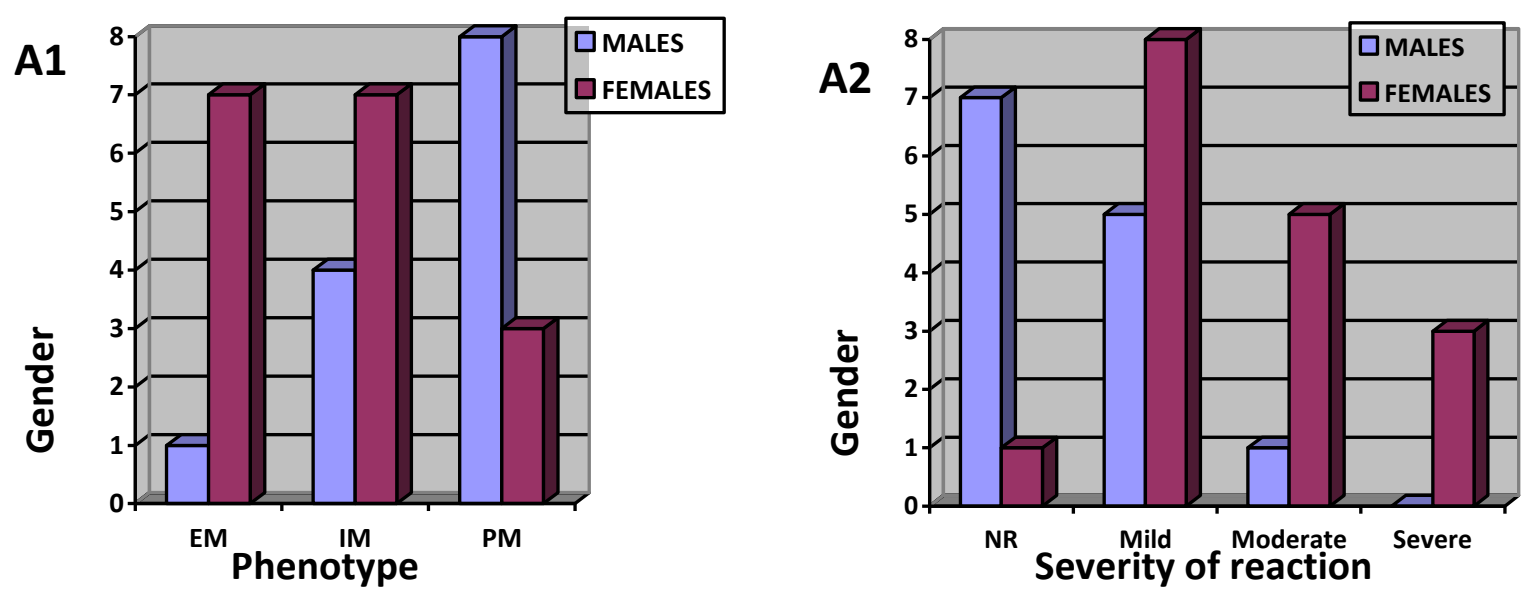

Figure 2. Chart showing the relationship between gender and (A1) phenotype, (A2) severity adverse reaction, experienced by participants. NR - No reaction

B1

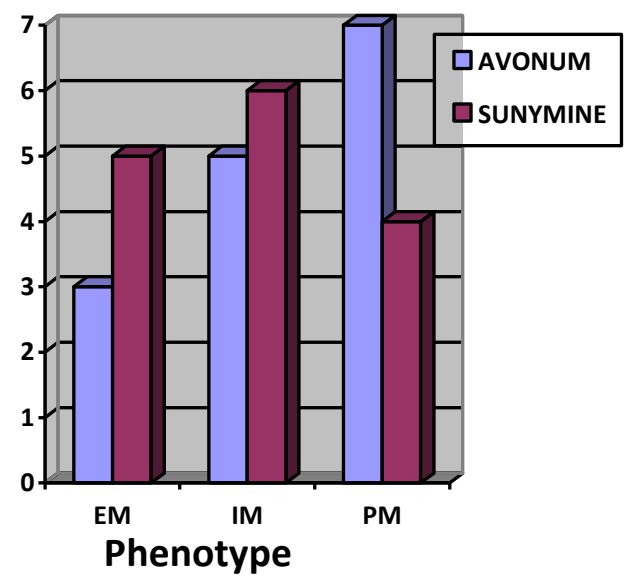

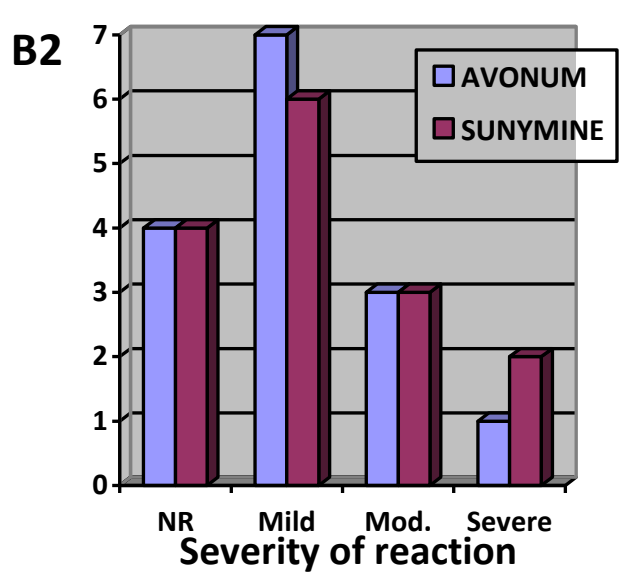

Figure 3. Relationship between the different brands used and (B1) Phenotype (B2) Severity of adverse reaction experienced by participants. 


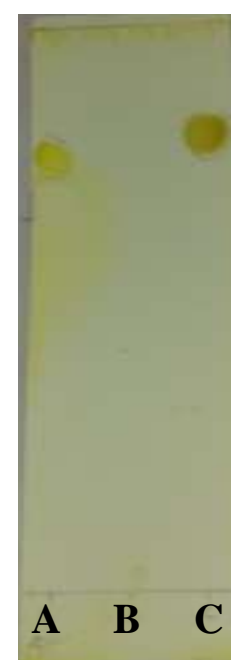

I

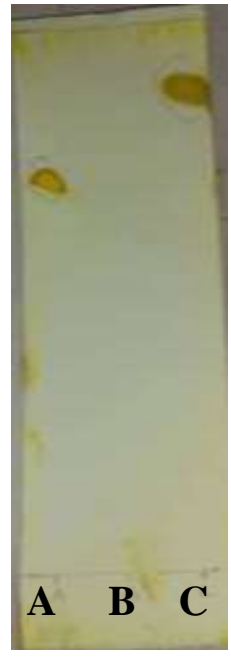

II

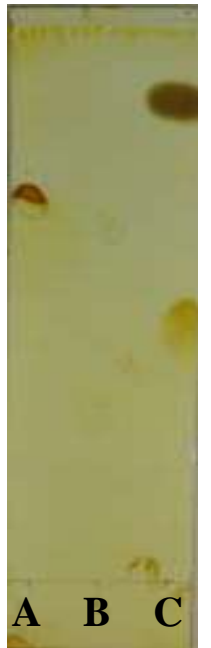

III
Figure 4. Representations of the TLC plates depicting (I: Extensive metabolizer. II: Intermediate Metabolizer and III: Poor metabolizer):

A: spots of test sample from post drug urine.

B: spots of test sample from blank urine.

C: spots of standard solution

\section{Discussion}

Thirty healthy volunteers participated in the survey, $56.67 \%$ (17) were females and $43.33 \%$ males. $33.33 \%$ were from Bayelsa, 20\% Rivers, $16.67 \%$ Delta, $13.33 \%$ Edo, 10\% Cross river and $6.67 \%$ Akwa Ibom. TLC analysis of drugs and drug metabolite expressed in biological fluids require a distinct knowledge of the standard $\mathrm{R}_{\mathrm{f}}$ values of the metabolites concerning the pure drug as well as the polarity of these samples. A drug is said to be metabolized if the polarity of the metabolites is higher than that of the pure drug otherwise, it is said to have been excreted unchanged. The degree of representative Rfs on the thin layer chromatogram shows that the more polar substances are better adsorbed on the stationary silica phase of the chromatogram than the non-polar drugs and as such requires a polar mobile phase to distinctively cause elution of the metabolite for the pure drugs. Hence the more polar metabolite shows a lesser $R_{f}$ value than the less polar drug sample (table 1 ). A report by Lynch, et al., suggested that CYP2D6 oxidative phenotyping across the globe suggests substantial variations amongst racial and inter-ethnic groups. They, however, depict no noticeable variation in the rate of promethazine metabolism amongst the various ethnic groups studied, thereby indicating similarity in the manner of CYP2D6 expression amongst the Niger delta population. ${ }^{12}$ Also, another report noted that individual with high muscles mass possesses a higher metabolic rate than an individual with less muscle mass. ${ }^{13}$ From the chromatogram intensities, $33.3 \%$ of the study population were poor metabolizers (IMs), $40 \%$ were intermediate metabolizers (IM), and $26.67 \%$ extensive metabolizers (EMs). Little or no elimination of $n$-desmethylpromethazine was observed for subjects with poor metabolism. $R_{f}$ values of $n$-desmethylpromethazine shows stipulated ranges in correspondence with the intensity of the spots. $R_{f}$ values of $0.66-0.70$ were implicated with spots of thicker intensities implying a poor rate of metabolism. Whereas, $0.74-0.77$ depicted less intense spots thus implying extensive metabolism. It is believed that the $\mathrm{R}_{\mathrm{f}}$ values of a drug when extensively metabolized are slightly lesser than the $R_{f}$ value of the same drug when poorly metabolize due to the extent of the polarity of the metabolite. Promethazine is also metabolized by CYP2B6 that has higher capacity but a lesser affinity. In some patients, spots were not detected at all, showing a clear demarcation from IM and the possibility of the drug not being rapidly metabolized. These individuals can be classed as ultra-rapid metabolizers. Hence, appropriate care should also be taken in the administration of drugs as toxicity is bound to occur. Drug individualization is preferred for such patients to minimize the risk of adverse reactions. About $40 \%$ of the study population were IMs. These 
individuals only requires a higher dose and are sometimes implicated as extensive metabolizers since their responses to drugs are not sufficiently different. The variation in spot intensities between the Ultra-rapid and EMs were so little that they could be classified as extensive metabolizers. This is many times, these two phenotypes are most regularly grouped. Extensive metabolizers experienced little or no adverse reactions after the single-dose therapy of promethazine. They had spot intensities to be less intense compared to those of IMs and PMs. These individuals thus express the normal activity of CYP2D6. The metabolites and degree of metabolism suggest the extent of optimal therapeutic response observed by the patient in pharmacogenomic studies.

\section{Conclusion}

Phenotyping is an explicitly useful method in the identification of oxidative polymorphism and genetic variation in individual treatment with drugs whose metabolism involves oxidative pathways. The use of thin-layer chromatography as a tool for phenotypic identification can adequately be used in determining different metabolizers in contemporary medicine. Having the phenotypic knowledge on the rate of drug metabolism will help in the clinical choices even in an emergency scenario as an individual from the same family may likely metabolize a giving drug in the same manner due to their genetic similarities.

\section{References}

1. David J. Promethazine, WOODS. 1990, Vol 2; page 10.

2. Elden Berla Thangam, Ebenezer Angel Jemima, Himadri Singh, Mirza Saqib Baig, Mahejibin Khan, Clinton B. Mathias, Martin K. Church, and Rohit Saluja. The Role of Histamine and Histamine Receptors in Mast Cell-Mediated Allergy and Inflammation: The Hunt for New Therapeutic Target. 2018, Vol. 9, page 1,2

3. Marcia L. Buck. Pediatric pharmacotherapy: promethazine recommendation for safe use in children. 2010, Vol 16(3).

4. Davidson AG. Ultraviolet-visible absorption spectrophotometry. In Beckett AH, Stenlake JB, ( $4^{\text {th }}$ edn), Practical Pharmaceutical chemistry. CBS Publishers and Distributors, New Delhi 2002, 275-278.

5. Ajibola A.O. Essential medicinal chemistry: Drugs acting on the peripheral nervous system: 3rd Ed. 2005, page (267-274).

6. Courtney C. Heather C. and James W. Oxidation of Selected Phenothiazine Drugs during Sample Preparation: Effects of Varying Extraction Conditions on the Extent of Oxidation. Journal of Analytical Toxicology. 2018, 42(2), Pages 99-114, https://doi.org/10.1093/jat/bkx067

7. Kiran Jadhav, Dinesh Dhamecha, Amol Tate, Harshad Tambe, and Mrityunjaya B. Patil. Application of UV spectrophotometric method for easy and rapid estimation of lafutidine in bulk and pharmaceutical formulation. 20112(4): 264-267. doi: 10.4103/2229-4708.93398

8. Marja-Liisa Dahl, Per Dalén, and Ayman Al-Shurbaji. Clinical relevance with focus on psychotropic drugs. Department of Molecular genetics of CYP2D6: Br J Clin Pharmacol. 2002; 53(2): 111-122.

9. Basavaiah K, Raghu M.S., and Vinay K.B. Simple and a rapid spectrophotometric assay of levocetirizine in pharmaceuticals through charge-transfer complexation using chloranilic acid and 2,3-dichloro-5,6-dicyanoquinone as $\pi$-acceptors. 2011, 26(3), page 319-328. DOI: http://dx.doi.org/10.4314/bcse.v26i3.

10. Taylor G., Houston J.B, Shaffer J., and Maweri G. Pharmacokinetics of promethazine and its sulphoxide metabolite after intravenous and oral administration to man. Journal of clinical pharmacy. 1983, (15) page 287-293 
11. Ebeshi U. Benjamin, Edebi N. Vaikosen and Kemetimiware T. Temes. High-Performance ThinLayer Chromatographic and Ultra-Violet spectrophotometric fingerprinting of some beta-lactam antibiotics. Journal of Science and Practice of Pharmacy. 2015; 2(1): 64-64. ISSN: 2449-0458.

12. Lynch T, Price A. The effect of cytochrome P450 metabolism on drug response, interactions, and adverse effects. American Family Physician. 2007, 76(3): 391-6. PMID 17708140.

13. Cantisani C, Ricci S, Grieco T, Paolino G, Faina V, Silvestri E, and Calvieri S. BioMed Research International: Topical Promethazine Side Effects: Our Experience and Review of the Literature. 2013 pages 1-9. 


\section{For your Research References Requirements, Always log on to www.sphinxsai.com}

\section{International Journal of PharmTech Research \\ International Journal of ChemTech Research}

